\title{
Mathematical and computational modeling of a ferrofluid deformable mirror for high-contrast imaging
}

\author{
Aaron J. Lemmer ${ }^{\mathrm{a}}$, Ian M. Griffiths ${ }^{\mathrm{b}}$, Tyler D. Groff ${ }^{\mathrm{a}}$, Andreas W. Rousing ${ }^{\mathrm{c}}$, and N. Jeremy \\ $\operatorname{Kasdin}^{\mathrm{a}}$ \\ ${ }^{a}$ Dept. of Mechanical \& Aerospace Engineering, Princeton University, Princeton, NJ USA \\ ${ }^{b}$ Mathematical Institute, University of Oxford, Oxford, England, UK \\ ${ }^{c}$ Dept. of Electrical Engineering, Technical University of Denmark, Lyngby, Denmark
}

\begin{abstract}
Deformable mirrors (DMs) are an enabling and mission-critical technology in any coronagraphic instrument designed to directly image exoplanets. A new ferrofluid deformable mirror technology for high-contrast imaging is currently under development at Princeton, featuring a flexible optical surface manipulated by the local electromagnetic and global hydraulic actuation of a reservoir of ferrofluid. The ferrofluid DM is designed to prioritize high optical surface quality, high-precision/low-stroke actuation, and excellent low-spatial-frequency performance - capabilities that meet the unique demands of high-contrast coronagraphy in a space-based platform. To this end, the ferrofluid medium continuously supports the DM facesheet, a configuration that eliminates actuator print-through (or, quilting) by decoupling the nominal surface figure from the geometry of the actuator array. The global pressure control allows independent focus actuation. In this paper we describe an analytical model for the quasi-static deformation response of the DM facesheet to both magnetic and pressure actuation. These modeling efforts serve to identify the key design parameters and quantify their contributions to the DM response, model the relationship between actuation commands and DM surface-profile response, and predict performance metrics such as achievable spatial resolution and stroke precision for specific actuator configurations. Our theoretical approach addresses the complexity of the boundary conditions associated with mechanical mounting of the facesheet, and makes use of asymptotic approximations by leveraging the three distinct length scales in the problem - namely, the low-stroke $(\sim \mathrm{nm})$ actuation, facesheet thickness $(\sim \mathrm{mm})$, and mirror diameter $(\sim \mathrm{cm})$. In addition to describing the theoretical treatment, we report the progress of computational multiphysics simulations which will be useful in improving the model fidelity and in drawing conclusions to improve the design.
\end{abstract}

Keywords: Deformable Mirror, Ferrofluid, Influence Function, Adaptive Optics, Wavefront Control, Coronagraphy, High-Contrast Imaging, Exoplanet

\section{BACKGROUND AND INTRODUCTION}

Ground-based observatories equipped with adaptive optics (AO) have pioneered the direct imaging of exoplanets and circumstellar disks. ${ }^{1,2}$ Freed from the performance-limiting aberrations induced by Earth's turbulent atmosphere, future space-borne telescopes with advanced AO-beginning with the upcoming WFIRST-AFTA mission - will enable discovery and investigation of potentially habitable exo-Earths. ${ }^{3-5}$ In order to maximize the number of characterizable planets, space-based AO must achieve a high degree of contrast between a target star and the region of space surrounding it, and this high-contrast search region must be as large as possible. Stellar coronagraphs designed to manage diffracted starlight suffer from optical aberrations, which create speckles that compromise the contrast over this field of view. Closed-loop wavefront control techniques, implemented with deformable mirrors, must suppress any quasi-static speckles that might obscure (or be indistinguishable from) a planet. 


\subsection{Rethinking AO for space-based coronagraphy: ferrofluid deformable mirror}

Deformable mirrors $(\mathrm{DMs})$ provide the mission-critical capability that these wavefront control routines need to achieve the extreme degree of speckle suppression required to image faint exo-Earths. In a recent study for a space-based coronagraph mission, detection of its faintest targeted planet at $4 \times 10^{-11}$ contrast demands that low-order modes of wavefront aberration be stabilized at the picometer level; ${ }^{6}$ precision requirements for broadband correction may be even more restrictive. ${ }^{7}$ Effecting such stringent wavefront stability requirements over a maximum search area means that the electric field must be controlled out to relatively high spatial frequencies.

Within the traditional AO framework, which has a heritage in ground-based AO where high actuator stroke and high speed capabilities take precedence, an AO system relies on a single DM (or at most two) to meet the exacting wavefront and wide field-of-view requirements. Due to these emphases, existing electrostrictive and capacitive DM technologies are designed to maximize speed, actuator density, and stroke; as a result, they are not always well matched to the space-based imaging problem. ${ }^{8}$ First, the control laws typically used in focal-plane wavefront control do not utilize the full range of stroke current DM devices offer. (Electric-field conjugation ${ }^{9}$ and stroke minimization ${ }^{10}$ achieve the target contrast with low actuator stroke - on the order of $\sim \lambda / 10$ or less. ${ }^{11}$ ) Second, for the sake of improved manufacturability and simplified control implementation, contemporary DM designs are fabricated with nominally flat reflective facesheets. Even with high actuator counts, nominally flat DMs cannot approximate low order shapes such as focus or tip-tilt without significant residual wavefront error in mid-spatial frequencies. Moreover, DMs with membrane- or thin plate-type facesheets are susceptible to imperfections in the optical surface in the vicinity of each actuator ${ }^{12,13}$ which introduce aberrations with periodic spatial structure to the optical wavefront, called actuator print-through or quilting. Although current manufacturing procedures can achieve surface figure in MEMS-type DMs of less than $10 \mathrm{~nm}$ RMS, the effect of this surface quality degradation still significantly limits performance. Since the periodicity of these features is correlated to the actuator pitch, the fundamental spatial frequency associated with quilting is twice the Nyquist frequency, and uncontrollable.

The ferrofluid DM described here (see Figure 1) is designed for operation in this high-precision low-stroke space-based regime. Actuator print-through is eliminated by providing a continuum support for the thin, flexible facesheet, eliminating the mechanical coupling between the optical surface quality and actuator location. A facesheet of this type may be uniformly polished and coated using well-established techniques to meet surface quality and reflectivity specifications. Although the actuator packing density in this ferrofluid-based device
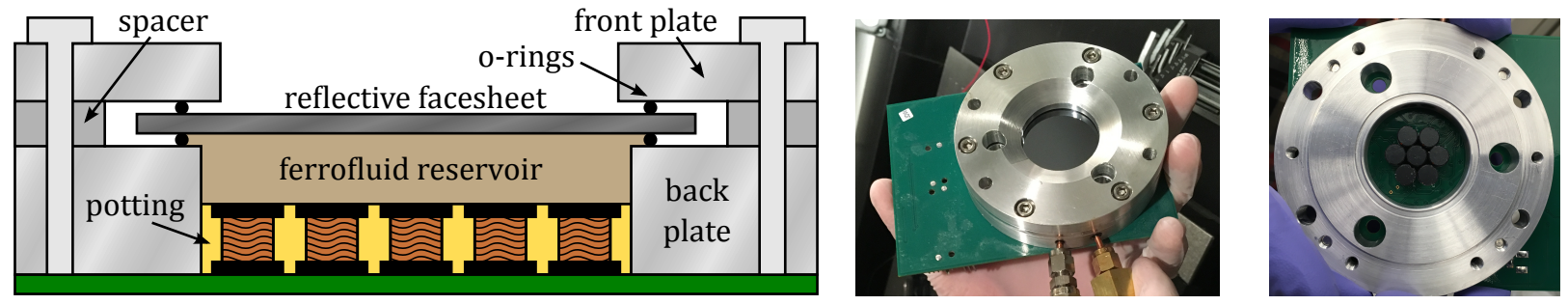

Figure 1: Cross-sectional schematic diagram of the ferrofluid DM (left, not to scale) and photograph of an operational 7-actuator prototype (center). In the prototype shown, the facesheet is mounted between a front bezel and a back plate, sealed and protected by a pair of o-rings, with compressive force fixed by a spacer plate. Beneath the ferrofluid reservoir and embedded in a potting material, an array of electromagnetic coils provides local control of the magnetic field within the fluid domain (right).

cannot match that of contemporary electrocapacitive or electrostrictive DMs, it possesses a means to tune its nominal optical power via pressure regulation. With the capacity to control nominal focus the DM is no longer restricted to collimated space, and as shown in Groff, et al., ${ }^{14}$ when used in conjunction with a high-actuatorcount DM, this enables an entirely new approach to AO system design in which nearly every optical surface is accessible by the closed-loop control law and may thus contribute to the system's overall corrective precision and capability. In this new paradigm, mid-spatial frequency errors are corrected via a series of lower-actuator-count DMs that provide controllability at each conjugate plane within the coronagraph, instead of relying on one or 
two high-risk, controllable surfaces. As a consequence, complicated aberrations can be more readily controlled all while re-distributing mission risk and lowering cost.

\subsection{Previous work with magnetic fluid deformable mirrors}

The modeling and design of the ferrofluid DM builds on the heritage of previous research on liquid-based mirror technologies for space-based astronomical AO or vision science applications. Such mirrors are based on the simple fact that the free liquid surface follows lines of equal potential. The simplest and oldest of these designs utilizes a rotating pool of mercury, which forms a parabolic shape having a focal length tunable via the angular speed of the turntable. ${ }^{15,16}$ An adaptation of this concept utilizes the mercury as a carrier fluid for ferromagnetic nanoparticles (creating a ferrofluid ${ }^{*}$ ), permitting transformation of the parabola into a sphere by application of an externally applied magnetic field. ${ }^{17}$ Another adaptation transmits an electric current through the mercury, allowing it to be deformed locally via an external magnetic field produced by an array of coils. ${ }^{18}$ All of these concepts have been investigated as candidate primary mirrors for large-aperture ground and space-based telescopes.

Although mercury's high reflectivity and low melting point make it a suitable liquid optical surface, its high density necessitates relatively large magnetic deforming forces when compared with water- or hydrocarbon-based ferrofluids. For magnetic-field manipulation by an array of electromagnetic coils, this translates directly to higher operating currents in the coils, and thus increased power consumption and ohmic heat dissipation in the mercury. A potential solution is to augment local magnetic actuation with permanent magnets on linear actuators.

Much effort has been invested into developing free-surface magnetic fluid DMs with lower-density carrier fluids, which have achieved peak-to-valley strokes approaching $50 \mu \mathrm{m}$ and $\sim 100 \mathrm{~nm}$ precision. ${ }^{19,20}$ The airferrofluid interface has poor optical reflectivity on its own, and a key enabling technological development of the free-surface magnetic fluid DM was the metal liquid-like film (MELLF) ${ }^{21}$ A MELLF is an extremely thin interfacial colloidal suspension of silver nanoparticles that can give a ferrofluid practical optical reflectivity. In addition to being physically impractical for zero-gravity applications, the reflectivity and spectral response of the MELLFs designed for free-surface ferrofluid DMs cannot provide sufficient and uniform throughput in broadband for high-performance coronagraphy, even on the ground. Free-surface ferrofluid mirrors are catered to the regime of lower wavefront quality but significantly higher stroke and are best suited for ground-based operation. For space-based AO, we seek to design a mirror with better wavefront quality and reflectivity, and low-stroke, high-precision actuators.

Despite the design elements which distinguish the ferrofluid DM studied here from previous liquid and magnetic fluid DMs, efforts to model the deflection response of those mirrors remains relevant and instructive. In particular, Iqbal and Ben Amara derived the governing equations for the dynamic response of a free-surface ferrofluid DM for retinal imaging applications in ophthalmic AO systems, and used the model to successfully aid in the design and simulate the control of the device. ${ }^{19}$ The authors treated nonlinearity and coupling in the system by applying a large and uniform linearizing magnetic field allowing them to solve for the response of their mirror using perturbation techniques. A linearizing uniform field is not applied to the ferrofluid DM studied here, in part because the large constant current required to operate the linearizing Maxwell coil increases power consumption and in part because the mode of pressure control would strongly affect the linearization. Nevertheless, because the ferrofluid DM operates in a regime of low stroke, asymptotic analysis offers a promising avenue for simplifying the model, as will be demonstrated below.

\subsection{This work}

In this paper a mathematical model is developed for the deformation of the ferrofluid DM facesheet to both pressure and magnetic actuation. In the future, such a model will be necessary in order to develop algorithms to command the facesheet deflection for high-performance wavefront control schemes. Mathematical modeling is also essential for understanding the physical principles and parameters that underpin the performance of the device. Due to the couplings between the state of stress within the ferrofluid, the electromagnetic state of the

*A ferrofluid, which differs significantly from a magnetorheological fluid in that it does not change viscosity under applied magnetic field, is a stable colloidal suspension of sub-domain ferromagnetic nanoparticles which behaves in the bulk as a paramagnetic medium. In traditional ferrofluids using water- or hydrocarbon-based carrier fluids, the particles are coated in a surfactant to prevent mutual attraction and aggregation. 
system, and the facesheet flexure, tuning of the design parameters is nonintuitive and the engineering process benefits from an informed physico-mathematical analysis. The modeling efforts in this paper will illuminate the fundamental importance of and interplay between key design parameters, such as the composition, geometry, and mechanical boundary conditions of the mounted facesheet; ferrofluid properties and volume; and actuator size, strength, and spacing.

The paper is organized as follows: in $\S 2$ the governing equations for the deflection of the ferrofluid DM facesheet are derived, carefully noting key assumptions. The derivation is presented component-wise, describing the solid mechanics of the facesheet in $\S 2.1$, the electromagnetic state within the ferrofluid in $\S 2.2$, and the ferrofluid behavior in $\S 2.3$. These components are assembled in $\S 2.4$, yielding the governing quasi-static equations. In $\S 3$ the system is non-dimensionalized, and the low-stroke regime of operation is utilized to reduce the complexity of the theory in an asymptotic analysis, which results in analytic solutions describing the facesheet deflection. The role of computational multiphysics in the development and improvement of the model fidelity is outlined in $\S 4$. Finally, the implications of the model are summarized in $\S 5$, and future steps in the modeling work are outlined.

\section{MATHEMATICAL MODEL FORMULATION}

In this section we seek a mathematical model to describe the deflection of the DM facesheet in response to actuation commands that are mechanically coupled through the ferrofluid. The model must describe the solid mechanics that determine the facesheet deflection profile due to the interfacial stress distribution from the ferrofluid, the electromagnetic state within the domain of the ferrofluid due an array of inductive coils, and the ferrofluid physics that mediates the interaction between these electromagnetic and solid-mechanical states. The facesheet of the ferrofluid DM is modeled as a horizontal laterally loaded plate, and its shape is determined as the deflection of the middle plane of the plate relative to its nominal unstressed state. The actuator is treated as an idealized point-dipole whose moment is computed from the coil characteristics. The interaction between the resulting magnetic field and the ferrofluid is described as a magnetically induced state of stress, and the surface traction resulting from this stress gives the load distribution on the facesheet from the ferrofluid.

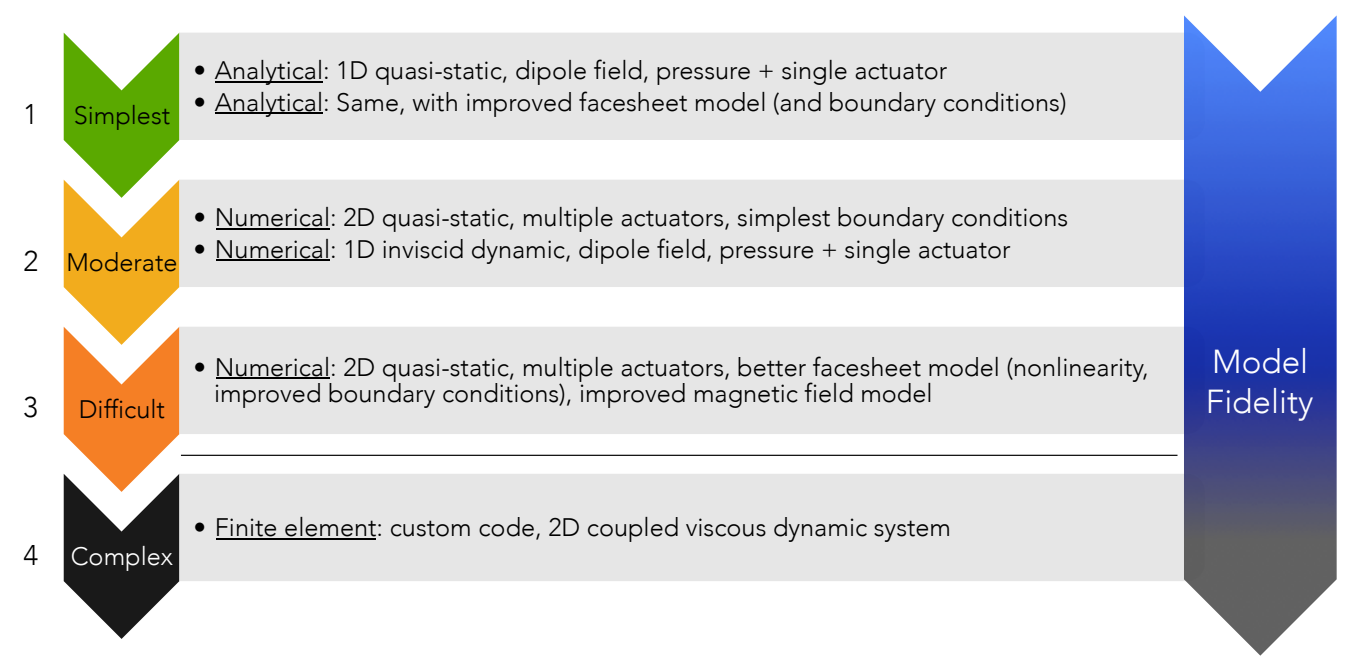

Figure 2: The model is developed incrementally, with each added complexity aimed at improving model fidelity. The simplest models are analytically solvable 1D quasi-static ODEs (1). When 2D results or dynamic analyses are demanded, the governing differential equations must be generalized to PDEs (2). Computational multiphysics can be implemented to model complex facesheet geometries and magnetic fields for improved fidelity (3), but a custom computational code (4) will likely be necessary for a full simulation of the $2 \mathrm{D}$ coupled system.

The primary challenges in the mathematical analysis of this system are correctly treating the strong coupling associated with the interface between the ferrofluid and solid facesheet and solving for the deflection of a moveable 
boundary. As shown in Figure 2, we build our model from a simple foundation, taking care to acknowledge the simplifying assumptions which may result in low model fidelity, in an attempt to circumvent any erroneous conclusions which may result from misinterpreting any nonintuitive behavior due to the coupling.

At the center of the model are two coupled partial differential equations describing the linear momentum balance of the fluid - modified to include magnetic effects - and the dynamic boundary condition at the facesheetferrofluid interface. The vertical deflection at any point on the facesheet surface can be ascertained by solving the second equation. The fundamental solution to this equation due to a single actuation source is defined as an influence function, which is a generalization of the Green's function. As long as the system behavior can be adequately described by linear differential equations, superposition can be exploited to describe the aggregate influence of many actuators on the facesheet. This is true in the analytical results presented here, where without loss of generality, it is sufficient to model the effect of only one magnetic actuator that has arbitrary coordinates. Whereas a model in cylindrical coordinates better represents a real optical device with circular symmetry, we employ Cartesian coordinates to simplify the derivation. The model is derived in two-dimensional Cartesian coordinates in sections $\S 2.1-\S 2.3$, and the $1 \mathrm{D}$ quasi-static governing equations assembled in $\S 2.4$ and analytically solved in $\S 3$.

\subsection{Solid mechanical description of the facesheet deflection}

The facesheet is modeled as a laterally loaded plate experiencing small deflections $w(x, y, t)$ and obeying the partial differential equation

$$
D \nabla^{2} \nabla^{2} w(x, y, t)=q(x, y, t)
$$

where $D$ is the flexural rigidity of the facesheet, $\nabla^{2}$ is the Laplacian operator, and the function $q(x, y, t)$ describes the stress distribution across the back of the facesheet due to the ferrofluid. ${ }^{22}$ The coordinates involved in this equation are defined and illustrated in Figure 3. Once the form of $q(x, y, t)$ is determined and inserted, the solution of this fourth-order differential equation provides the boundary condition for the total stress within the fluid domain.

In deriving (1) it is assumed, firstly, that the middle plane of the facesheet is the neutral surface and experiences zero strain. Practically, this implies that the boundary conditions on the plate only describe reaction forces normal to the facesheet; the edges of the facesheet are free to move in the plane of the plate. These assertions are valid so long as the strain in the middle plane is small relative to the maximum bending strain, which is true when the deflections are small relative to the facesheet thickness. Secondly, we assume deflections in the facesheet are small enough to warrant small-angle approximations in the local radius of curvature, which is generally true under the foregoing assumptions. A direct consequence is that the unit normal vector to the facesheet surface is approximately vertical, $\hat{\mathbf{n}} \| \hat{\mathbf{z}}$, and thus $q(x, y, t)$ acts in the $z$-direction only. Finally, we assume that the effect on bending of the shear forces and the local compressive stress produced by the ferrofluid are neglected in determining the relationship between bending moment and curvature. In short, these forces contribute significantly to the bending moments when the facesheet thickness is significant, so the error in the predicted facesheet deflection is minimal when its thickness is small relative to the ferrofluid DM aperture.

Considering that the ferrofluid DM is intended for employment in a high-contrast wavefront control scheme where strokes are typically less than $\sim \lambda / 10$ at optical wavelengths, these significant simplifying assumptions are very compelling, as shown in Table 1.

Typical Ferrofluid DM Dimensions

\begin{tabular}{lcc}
\hline Parameter & Variable & Scale \\
\hline Facesheet deflection (stroke) & $w(x, y, t)$ & $\lesssim 50 \mathrm{~nm}$ \\
Facesheet thickness & $h$ & $\sim 500 \mu \mathrm{m}$ \\
Mirror aperture & $a$ & $\sim 5 \mathrm{~cm}$ \\
\hline
\end{tabular}

Table 1: For typical magnitudes of ferrofluid DM dimensions, $h / a \sim 10^{-3}$. For the space-based wavefront control regime, we expect $w / a \lesssim 10^{-6}$ and $w / h \lesssim 10^{-4}$. 


\subsubsection{Mechanical boundary conditions}

For boundary conditions on $w$ we assume the facesheet edges are uniformly fixed. For a rectangular mirror with aperture size $a_{x} \times a_{y}$, this implies

$$
\left.w\right|_{x=0, a_{x}}=\left.w\right|_{y=0, a_{y}}=0 \quad \text { and }\left.\quad \frac{\partial w}{\partial x}\right|_{x=0, a_{x}}=\left.\frac{\partial w}{\partial y}\right|_{y=0, a_{y}}=0
$$

This is highly idealized, since the o-ring compression which holds the facesheet in place has some inherent flexibility. In reality, the o-ring compression is not perfectly uniform and introduces some combination of vertical and horizontal reaction forces along with reaction moments, and the latter two are neglected in the simplest facesheet model assumptions above.

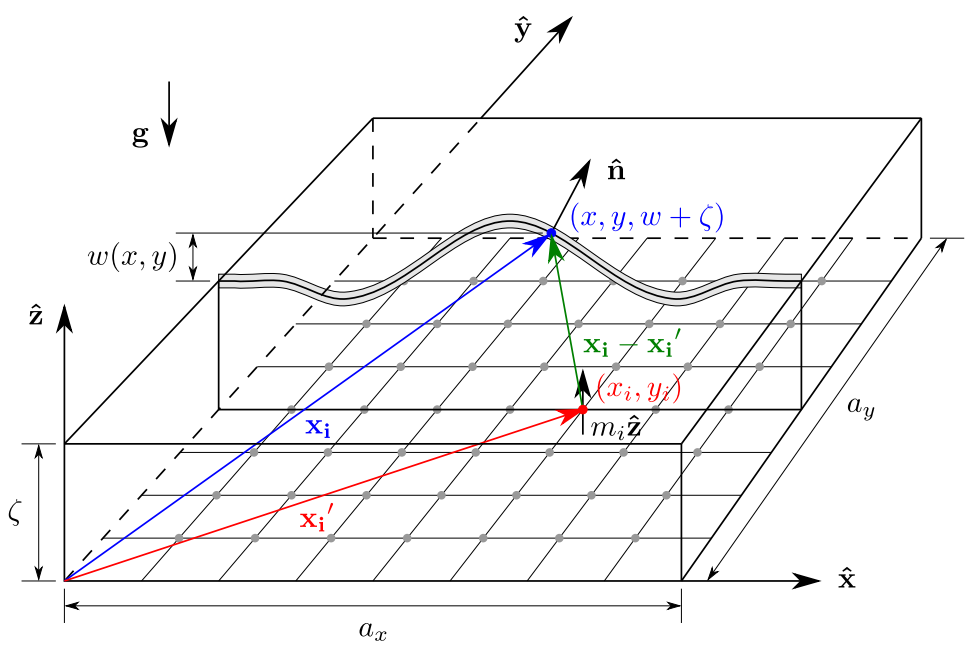

Figure 3: Cross-sectional diagram identifying the variables in the solid-mechanical and electromagnetic descriptions. The vertical displacement of the neutral surface of the facesheet is denoted by $w$.

\subsection{Electromagnetic description}

The magnetization of a ferrofluid is generally nonlinear and it can be modeled in terms of the magnetic susceptibility and the magnetic field as $\mathbf{M}=\chi_{m} \mathbf{H}^{*}$ where $\chi_{m}=\partial M / \partial H$. The initial, linear susceptibility $\chi_{0}=M / H$ may be found as $\lim _{H \rightarrow 0} \chi_{m}(H)$. A constitutive relation for the relationship between the magnetic induction and the magnetic field that captures the paramagnetic behavior of a ferrofluid is

$$
\mathbf{B}=\mu_{0}\left(1+\chi_{0}\right) \mathbf{H}=\mu \mathbf{H}
$$

The relevant geometry and variable definitions for describing the magnetic field within the ferrofluid domain is presented in Figure 3. The influence and orientation of an inductive coil located at $\mathbf{x}_{\mathbf{i}}{ }^{\prime}=x_{i} \hat{\mathbf{x}}+y_{i} \hat{\mathbf{y}}{ }^{\dagger}$ with the coordinates $\left(x_{i}, y_{i}\right)$, is described by the dipole moment vector $\mathbf{m}_{\mathbf{i}}=m_{i}(t) \hat{\mathbf{z}}$. The location and orientation of each coil is fixed within the array, and its dipole moment magnitude $m_{i}(t)$ is set by the coil's geometry, self-inductance, core permeability, and the average current commanded through it, $\bar{I}(t)$, which may change temporally.

The magnetic field produced by such a point-dipole coil is given by the curl of a dipole vector potential, ${ }^{23}$

$$
\mathbf{H}\left(\mathbf{x}_{\mathbf{i}}, t\right)=\frac{\mu_{0}}{4 \pi \mu} \nabla \times \frac{\mathbf{m}_{\mathbf{i}} \times\left(\mathbf{x}_{\mathbf{i}}-\mathbf{x}_{\mathbf{i}}{ }^{\prime}\right)}{\left|\mathbf{x}_{\mathbf{i}}-\mathbf{x}_{\mathbf{i}}\right|^{3}},
$$

*Throughout this paper, all vector and tensor quantities are indicated in bold typeface. Wherever the letter ' $\mathrm{m}$ ' appears in a subscript, bold or italic, the designation distinguishes a magnetic quantity.

${ }^{\dagger}$ The primed notation is used for vectors identifying the location of a field source relative to the origin, whereas the unprimed version denotes the location at which a field is sampled, relative to the origin. 
where the constitutive relation (3) has been used. In (4) the location at which the field is sampled is $\mathbf{x}_{\mathbf{i}}=$ $x \hat{\mathbf{x}}+y \hat{\mathbf{y}}+(w+\zeta) \hat{\mathbf{z}}$ and $\left|\mathbf{x}_{\mathbf{i}}-\mathbf{x}_{\mathbf{i}}{ }^{\prime}\right|^{2}=\left(x-x_{i}\right)^{2}+\left(y-y_{i}\right)^{2}+(w+\zeta)^{2}$ is the square of the distance from the electromagnet to the measurement point. Evaluating (4), the magnetic field due to a single coil actuator is

$$
\mathbf{H}\left(\mathbf{x}_{\mathbf{i}}, t\right)=\frac{\mu_{0} m_{i}(t)}{4 \pi \mu} \frac{3\left(x-x_{i}\right)(w+\zeta) \hat{\mathbf{x}}+3\left(y-y_{i}\right)(w+\zeta) \hat{\mathbf{y}}+\left[2(w+\zeta)^{2}-\left(x-x_{i}\right)^{2}-\left(y-y_{i}\right)^{2}\right] \hat{\mathbf{z}}}{\left[\left(x-x_{i}\right)^{2}+\left(y-y_{i}\right)^{2}+(w+\zeta)^{2}\right]^{5 / 2}} .
$$

\subsection{Ferrohydrodynamic description}

The fluid mechanical behavior in a ferrofluid system is governed by the Navier-Stokes equations with modifications to handle the interaction with a magnetic field. ${ }^{24}$ These modifications are fundamentally manifest in the state of stress within the fluid. Assuming incompressibility and denoting the velocity field as $\mathbf{u}=u_{x} \hat{\mathbf{x}}+u_{y} \hat{\mathbf{y}}+u_{z} \hat{\mathbf{z}}$, the equations for conservation of mass and linear momentum for a unit control volume are

$$
\begin{aligned}
\boldsymbol{\nabla} \cdot \mathbf{u} & =0 \text { and } \\
\rho\left[\frac{\partial \mathbf{u}}{\partial t}+(\mathbf{u} \cdot \boldsymbol{\nabla}) \mathbf{u}\right] & =\rho \mathbf{g}-\nabla p+\eta \nabla^{2} \mathbf{u}+\mathbf{f}_{\mathbf{m}},
\end{aligned}
$$

respectively. From left to right, the terms on the right-hand side of (7) are the gravitational body force per unit volume, where $\rho$ is the density and $\mathbf{g}$ is the gravitational acceleration; the net hydrodynamic and viscous forces arising from the Cauchy stress $\mathbf{T}$, where $p$ is the pressure and $\eta$ is the fluid shear viscosity; and $\mathbf{f}_{\mathbf{m}}$ is the magnetic body force per unit volume. The origin, form, and consideration of this final magnetic body force term, $\mathbf{f}_{\mathbf{m}}$, is the subject of the remainder of this section and $\S 2.3 .2$.

The magnetic stress tensor in a ferrofluid, $\mathbf{T}_{\mathbf{m}}$, is conventionally used to determine the magnetic body force $\mathbf{f}_{\mathbf{m}}=\boldsymbol{\nabla} \cdot \mathbf{T}_{\mathbf{m}}$ and the interfacial surface traction, $\mathbf{t}_{\hat{\mathbf{n}}}=\hat{\mathbf{n}} \cdot\left(\mathbf{T}+\mathbf{T}_{\mathbf{m}}\right) \cdot{ }^{24}$ Neglecting magnetostrictive effects due to incompressibility,

$$
\mathbf{T}_{\mathbf{m}}=-\left(p_{m}+\frac{1}{2} \mu_{0} \mathbf{H} \cdot \mathbf{H}\right) \mathbf{I}+\mu \mathbf{H} \mathbf{H},
$$

where the fluid-magnetic pressure is defined as $p_{m}=\mu_{0} \int_{0}^{H} M d H$. The magnetic body force on a fluid element is

$$
\mathbf{f}_{\mathbf{m}}=-\boldsymbol{\nabla} p_{m}+\frac{1}{2} \mu_{0} \chi_{0} \boldsymbol{\nabla}(\mathbf{H} \cdot \mathbf{H}) .
$$

\subsubsection{Kinematic boundary conditions}

The following kinematic condition relates the dynamic deflection of the facesheet at a point $z=\zeta+w(x, y, t)$ to the motion of the adjacent ferrofluid:

$$
u_{z}=\frac{\partial w}{\partial t}+u_{x} \frac{\partial w}{\partial x}+u_{y} \frac{\partial w}{\partial y} .
$$

On the immovable boundaries of the fluid reservoir, no-slip and no-penetration conditions are applied. These are

$$
u_{x}=u_{y}=u_{z}=0 \quad \text { on } \quad x=y=z=0, x=a_{x}, \text { and } y=a_{y} .
$$

\subsubsection{Dynamic boundary conditions on stress}

So far in $\S 2.3$, the interaction between the ferrofluid and an applied magnetic field (such as that defined in $\S 2.2)$ have been described. To complete the picture, it remains to link this physics with the description of the facesheet deflection from $\S 2.1$. This linkage is the dynamic boundary condition at the interface between the mirror facesheet and the ferrofluid, which balances the normal and tangential components of stress. As noted in $\S 2.1$, the interface is assumed to be the neutral plane of the plate, which experiences zero strain. Since the strain is in the elastic regime, the stress is zero tangent to this plane. Together with the no-slip condition on the ferrofluid at the facesheet, this implies the tangential interfacial stress is trivially continuous and zero.

In the vertical direction, (1) describes this balance. The total interfacial stress distribution $q(x, y, t)$ is determined by calculating the difference in the normal surface traction, $t_{\hat{n} \hat{n}}=\mathbf{t}_{\hat{\mathbf{n}}} \cdot \hat{\mathbf{n}}$, across the boundary. When magnetizable media are involved, there is generally a discontinuity in stress. Calculating this difference in surface 
traction accounts for the boundary conditions on $\mathbf{B}$ and $\mathbf{H}$ from Maxwell's equations, and reveals that a magnetic stress also exists, in addition to the fluid-magnetic pressure due to the magnetic body force. sSince the stress distribution on the facesheet acts in the $z$-direction owing to small-angle approximations, as stated in $\S 2.1, \hat{\mathbf{n}} \| \hat{\mathbf{z}}$. Thus $\hat{\mathbf{n}}=\hat{\mathbf{n}}_{\mathbf{1}}=-\hat{\mathbf{z}}$ for the ferrofluid medium and $\hat{\mathbf{n}}=\hat{\mathbf{n}}_{\mathbf{2}}=\hat{\mathbf{z}}$ for the facesheet medium. The normal surface traction on each side of the interface is

$$
\begin{aligned}
& t_{\hat{n} \hat{n}}^{(1)}=\hat{\mathbf{n}}_{\mathbf{1}} \cdot\left(\mathbf{T}+\mathbf{T}_{\mathbf{m}}\right) \cdot \hat{\mathbf{n}}_{\mathbf{1}}=-p+2 \eta \frac{\partial u_{z}}{\partial z}-p_{m}+\frac{1}{2} \mu_{0}\left(H_{1, z}^{2}-H_{1, t}^{2}\right)+\mu H_{1, z}^{2} \quad \text { and } \\
& t_{\hat{n} \hat{n}}^{(2)}=\hat{\mathbf{n}}_{\mathbf{2}} \cdot \mathbf{T}_{\mathbf{m}} \cdot \hat{\mathbf{n}}_{\mathbf{2}} \quad=\frac{1}{2} \mu_{0}\left(H_{2, z}^{2}-H_{2, t}^{2}\right)+\mu H_{2, z}^{2},
\end{aligned}
$$

where the subscripts $z$ and $t$ denote the normal and tangential components of the vectors, respectively. The traction in the ferrofluid medium is given by (12), and (13) reflects that in a non-magnetizable solid medium $\mathbf{M}=\mathbf{0}$ and $\mathbf{T}=\mathbf{0}$. The distributed load on the facesheet is thus

$$
q(x, y, t)=t_{\hat{n} \hat{n}}^{(2)}-t_{\hat{n} \hat{n}}^{(1)}=p+p_{m}-2 \eta \frac{\partial u_{z}}{\partial z}+\frac{1}{2} \mu_{0} \chi_{0}^{2}(\mathbf{H} \cdot \hat{\mathbf{z}})^{2},
$$

where $q$ is positive in the positive $z$-direction. The dynamic boundary condition on the normal component of the stress can be finally written as

$$
D \nabla^{2} \nabla^{2} w(\mathbf{u} ; x, y, t)=p+p_{m}-2 \eta \frac{\partial u_{z}}{\partial z}+\frac{1}{2} \mu_{0} \chi_{0}^{2}(\mathbf{H} \cdot \hat{\mathbf{z}})^{2} .
$$

This equation is analogous to the interfacial stress balance between two immiscible fluids, where the capillary pressure term (dependent on the second-order curvature expression) has been replaced with fourth-order expression representing plate deformation. The orientations of the constituent terms of the boundary condition are illustrated in Figure 4. In (15), the coupling between the facesheet deflection and the ferrofluid velocity profile has been established explicitly.

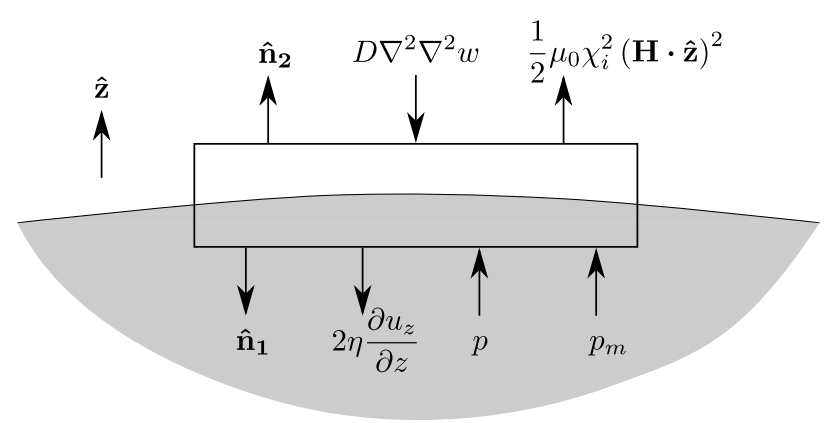

Figure 4: Balance of stresses on and orientation of unit vectors relative to a small surface element. Region 1 (grey) is the ferrofluid, and region 2 is the facesheet.

\subsection{Assembly of the quasi-static governing equations}

The solution to the dynamic boundary condition given by (15) is the deflection function $w(x, y, t)$ describing the deformed shape of the ferrofluid DM facesheet. When determined for a particular actuator, $w(x, y, t)$ is the influence function of that actuator. Equation 15 is coupled to the fluid flow through (6) and (7). It is explicitly subject to the mechanical, kinematic, and fluid dynamical boundary conditions given by (2), (10), and (11), and implicitly subject to the boundary conditions on the electromagnetic fields. Although the dynamic problem has been fully described here, it is beyond the scope of this paper to solve it. As described above, we begin with the simplest model - the one-dimensional quasi-static case - so as to seek an analytical solution for the influence functions.

We find the 1D quasi-static equations by taking the steady-state limit of the linear momentum conservation equation (7) and the kinematic boundary condition (10). In this limit all remaining explicit instances of the 
fluid velocity profile are proportional to $\eta$; as with other approaches in the literature, we also neglect the effect of viscous forces. ${ }^{19,24}$ The steady-state limit permits a non-zero fluid velocity, so the inviscid approximation is mathematically a very attractive step because it uncouples the flow field from the deflection given by (15).* Taken together, these simplifications reduce the model to the following two equations,

$$
\begin{aligned}
\mathbf{0} & =\rho \mathbf{g}-\nabla p+\mathbf{f}_{\mathbf{m}} \text { and } \\
D \nabla^{2} \nabla^{2} w(x) & =p+p_{m}+\frac{1}{2} \mu_{0} \chi_{0}^{2}(\mathbf{H} \cdot \hat{\mathbf{z}})^{2},
\end{aligned}
$$

where (9) has been substituted, and where (16) is essentially a constitutive equation for the pressure $p$ and its $z$-component is the only part that couples to (17).

For a ferrofluid at a practical operating temperature, it can be shown that $\mathbf{f}_{\mathbf{m}}$ is identically zero, ${ }^{24}$ implying by (9) that

$$
\boldsymbol{\nabla} p_{m}=\frac{1}{2} \mu_{0} \chi_{0} \boldsymbol{\nabla}(\mathbf{H} \cdot \mathbf{H}) \text {. }
$$

This does not imply that an applied magnetic field has zero effect on the ferrofluid; rather, the magnetic effect appears in the boundary condition on the fluid domain. Integrating the vertical component of (16) with $\mathbf{g}=-g \hat{\mathbf{z}}$ and evaluating at the facesheet, $z=\zeta+w(x)$, yields the hydrostatic expression $p=-\rho g w(x)+p_{0}$, where $p_{0}$ is the nominal pressure in the fluid at $z=\zeta$. Substituting this, along with the integral of (18) evaluated at the facesheet, into (17) yields

$$
D \nabla^{2} \nabla^{2} w\left(x ; p, m_{i}\right)+\rho g w\left(x ; p, m_{i}\right)=p+\frac{1}{2} \mu_{0} \chi_{0} \mathbf{H} \cdot \mathbf{H}+\frac{1}{2} \mu_{0} \chi_{0}^{2}(\mathbf{H} \cdot \hat{\mathbf{z}})^{2},
$$

where the magnetic field at the facesheet is given by (5) for the $i^{\text {th }}$ actuator and the subscript on $p_{0}$ has been dropped. This fourth-order ordinary differential equation describes the facesheet deflection profile along one dimension, and it is parameterized by the tunable reservoir pressure and the current-controllable dipole moment.

\section{ANALYSIS OF THE QUASI-STATIC GOVERNING EQUATION}

Before solving equation (19) for a general deflection profile or an influence function, we non-dimensionalize it using

$$
x=x_{c} X, \quad p=p_{c} P, \quad m_{i}=m_{c} M_{i}, \quad \text { and } \quad w\left(x ; p, m_{i}\right)=w_{c} W\left(X ; P, M_{i}\right),
$$

where $x_{c}$ is a characteristic length across the facesheet, $p_{c}$ is a characteristic pressure, $m_{c}$ is a characteristic electromagnet dipole moment, and $w_{c}$ is a characteristic facesheet deflection, describing the balance between the applied magnetic field and the rigidity of the mirror facesheet. Taking the logical values for the characteristic length, pressure, and dipole moment to be, respectively, the dimension of the mirror aperture, the nominal pressure, and the maximum operating dipole moment, then

$$
x_{c}=a, \quad p_{c}=p_{0}, \quad m_{c}=m_{\max }, \quad \text { and } \quad w_{c}=\frac{\mu_{0} \chi_{0} m_{\max }^{2}}{32 \pi^{2}\left(1+\chi_{0}\right)^{2} D a^{2}} .
$$

Substituting these expressions along with (5) into (19) yields

$$
\frac{\partial^{4} W}{\partial X^{4}}+4 \beta^{4} W=\psi P+M_{i} \frac{9\left(X-X_{i}\right)^{2}(\varepsilon W+\delta)^{2}+\left(1+\chi_{0}\right)\left[2(\varepsilon W+\delta)^{2}-\left(X-X_{i}\right)^{2}\right]^{2}}{\left[\left(X-X_{i}\right)^{2}+(\varepsilon W+\delta)^{2}\right]^{5}} .
$$

There are six dimensionless parameters which characterize (22):

$$
X_{i}=\frac{x_{i}}{a}, \quad \beta^{4}=\frac{\rho g a^{4}}{4 D}, \quad \psi=\frac{p_{0} a^{4}}{w_{c} D}, \quad \delta=\frac{\zeta}{a}, \quad \text { and } \quad \varepsilon=\frac{w_{c}}{a} .
$$

*Although validated experimentally for free-surface ferrofluid DMs, the inviscid flow approximation deserves further scrutiny for a fully-contained ferrofluid mirror with a membrane or plate facesheet because it is in the boundary layer near rigid walls (such as near the facesheet) that viscous effects can become significant. If viscous effects cannot be neglected, even the 1D quasi-static coupled problem becomes more intensive to solve. In the remainder of this paper, inviscid flow is assumed with this caveat in mind. 
The value $X_{i}$ is the non-dimensional $1 \mathrm{D}$ coordinate of the $i^{\text {th }}$ actuator. The parameter $\beta$ is functionally like an Eötvös or Bond number, and it describes the relative importance of the stress in the facesheet and the body forces acting on fluid elements. Its effect on the deflection is illustrated in the next section. The quantity $\psi$ scales the tunable pressure of the ferrofluid in the reservoir. The parameter $\varepsilon$ describes the aspect ratio, or smallness, of the facesheet deflection relative to the ferrofluid DM aperture, whereas $\delta$ describes a second aspect ratio of the ferrofluid reservoir depth relative to the mirror radius. Prototypical values of these parameters are presented in Table 2.

Prototype Ferrofluid DM Quantities

\begin{tabular}{lcc|lcc}
\hline Characteristic Dimension & Variable & Value & Dimensionless Parameter & Variable & Value \\
\hline Facesheet thickness & $h$ & $100 \mu \mathrm{m}$ & Bond number & $\beta$ & $3.59 \times 10^{-2}$ \\
Facesheet deflection & $w_{c}$ & $3.90 \times 10^{-11} \mathrm{~m}$ & Pressure scalar & $\psi$ & $3.65 \times 10^{11}$ \\
Mirror aperture & $a$ & $3.81 \times 10^{-2} \mathrm{~m}$ & Fluid depth aspect ratio & $\delta$ & 0.170 \\
Dipole moment & $m_{\max }$ & $2.61 \times 10^{-4} \mathrm{~A} / \mathrm{m}^{2}$ & Deflection aspect ratio & $\varepsilon$ & $1.02 \times 10^{-9}$ \\
\hline
\end{tabular}

Table 2: Prototypical quantities for a ferrofluid DM. Note that $w_{c}$ is a scaling parameter that does not alone predict the true deflection magnitude of an influence function; it must be multiplied by the dimensionless solution to $(22)$.

Equation 22 is subject to the dimensionless form of the mechanical boundary conditions first given by (2):

$$
\left.W\right|_{X=0,1}=0 \quad \text { and }\left.\quad \frac{d W}{d X}\right|_{X=0,1}=0 .
$$

In the following sections, (22) will be solved analytically subject to (24), in the presence of pressure actuation only. An asymptotic form of (22) will be used to find the facesheet deflection in the presence of both magnetic and pressure actuation and compared to a numerical solution to the full form of the equation.

\subsection{Exact solution with pressure actuation}

The solution to (22) in the absence of an applied magnetic field predicts the facesheet deflection in response to hydraulic control of the ferrofluid reservoir pressure. This solution can be utilized to calculate the sag and focal length of the optic. Furthermore, when local magnetic actuation is not utilized, the solution can be used as a baseline indicator for the accuracy of the solid-mechanical model - namely the accuracy of the predicted deflection magnitude and the validity of the boundary conditions and assumptions outlined in $\S 2.1$. Upon applying the dimensionless boundary conditions (24), the solution to (22) with $M_{i}=0$ is found to be

$$
\begin{aligned}
W(X ; P)=\frac{\psi P}{4 \beta^{4}}[1 & -\frac{\cosh \beta \sin \beta-\cos \beta \sinh \beta}{(\cos \beta+\cosh \beta)(\sin \beta+\sinh \beta)} \cosh (\beta+\beta X) \cos (\beta-\beta X) \\
& -\frac{\cosh \beta \sin \beta+\cos \beta \sinh \beta}{(\cos \beta+\cosh \beta)(\sin \beta+\sinh \beta)} \sinh (\beta+\beta X) \sin (\beta-\beta X) \\
& +\frac{\cos \beta \sinh \beta+\cosh \beta(\sin \beta+2 \sinh \beta)}{(\cos \beta+\cosh \beta)(\sin \beta+\sinh \beta)} \cosh \beta X \cos \beta X \\
& \left.+\frac{\cosh \beta \sin \beta-(\cos \beta+2 \cosh \beta) \sinh \beta}{(\cos \beta+\cosh \beta)(\sin \beta+\sinh \beta)} \sinh \beta X \sin \beta X\right] .
\end{aligned}
$$

This solution is shown, parameterized by pressure and Bond number, in Figure 5. We observe in Fig. 5b that the deflection increases with decreasing Bond number. Examining the expressions in (20), we see that for a mirror in which the interfacial stress dominates over gravity, the ferrofluid mirror strokes will be higher, as expected. 


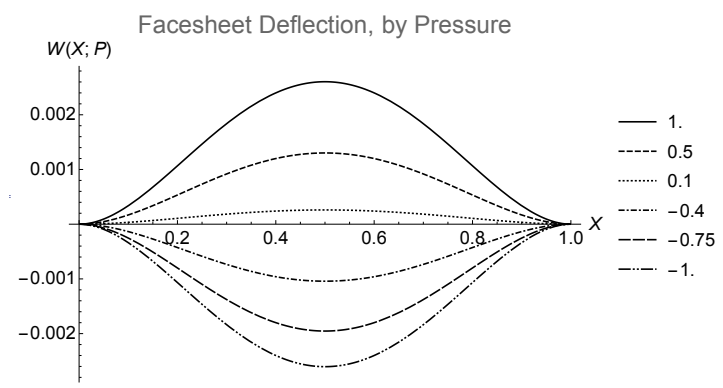

(a)

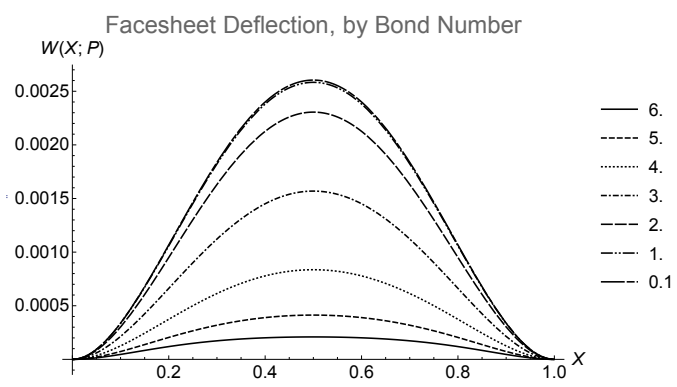

(b)

Figure 5: The analytical dimensionless facesheet deflection $W(X ; P)$ as determined by (25) is shown (a) for fixed Bond number and varied $P$ and (b) parameterized by Bond number $\beta$ with $P=1$.

\subsection{Asymptotic solution with pressure and a single magnetic actuator}

The smallness of the facesheet deflections typically used in focal-plane wavefront control schemes can be exploited in an asymptotic analysis to further simplify the dimensionless model with minimal error. Referring to Table 2, prototypical values for the aspect ratios described in the foregoing section are $\delta \sim 10^{-1}$ and $\varepsilon \sim 10^{-12}$, so it is expected that $\varepsilon \lll \delta<1$. In an effort to explore its asymptotic behavior, (22) is taken in the limit where $\varepsilon \rightarrow 0$ :

$$
\frac{\partial^{4} W}{\partial X^{4}}+4 \beta^{4} W=\psi P+M_{i} \frac{9\left(X-X_{i}\right)^{2} \delta^{2}+\left(1+\chi_{0}\right)\left[2 \delta^{2}-\left(X-X_{i}\right)^{2}\right]^{2}}{\left[\left(X-X_{i}\right)^{2}+\delta^{2}\right]^{5}} .
$$

The solution to (26) when including the inhomogeneous term corresponding to the effect of the $i^{\text {th }}$ actuator may be found via the method of variation of parameters. Defining the following equations to allow for a more compact representation,

$$
\begin{aligned}
& f_{1}(X)=\cosh (\beta+\beta X) \cos (\beta-\beta X) \\
& f_{2}(X)=\sinh (\beta+\beta X) \sin (\beta-\beta X) \\
& f_{3}(X)=\cosh \beta X \cos \beta X \\
& f_{4}(X)=\sinh \beta X \sin \beta X \\
& f_{5}(X)=\frac{9\left(X-X_{i}\right)^{2} \delta^{2}+\left(1+\chi_{0}\right)\left[2 \delta^{2}-\left(X-X_{i}\right)^{2}\right]^{2}}{\left[\left(X-X_{i}\right)^{2}+\delta^{2}\right]^{5}},
\end{aligned}
$$

the solution is

$$
\begin{aligned}
W\left(X ; P, M_{i}\right)= & \frac{\psi P}{4 \beta^{4}}+A f_{1}(X)+B f_{2}(X)+C f_{3}(X)+D f_{4}(X)+\frac{M_{i}}{8 \beta^{3}}[ \\
& -f_{1}(X) \int_{0}^{X}\left[f_{3}(u)(-\cosh \beta \sin \beta+\cos \beta \sinh \beta)-f_{4}(u)(\cosh \beta \sin \beta+\cos \beta \sinh \beta)\right] f_{5}(u) d u \\
& +f_{2}(X) \int_{0}^{X}\left[-f_{3}(u)(\cosh \beta \sin \beta+\cos \beta \sinh \beta)+f_{4}(u)(\cosh \beta \sin \beta-\cos \beta \sinh \beta)\right] f_{5}(u) d u \\
& +f_{3}(X) \int_{0}^{X}[\cosh \beta u \sin [\beta(-2+u)]-\cosh [\beta(2+u)] \sin \beta u \\
& \quad-\cos [\beta(-2+u)] \sinh \beta u+\cos \beta u \sinh [\beta(2+u)]] f_{5}(u) d u \\
& +f_{4}(X) \int_{0}^{X}[\cosh \beta u \sin [\beta(2-u)]+\cosh [\beta(2+u)] \sin \beta u \\
& \left.\quad-\cos [\beta(-2+u)] \sinh \beta u+\cos \beta u \sinh [\beta(2+u)] f_{5}(u) d u\right]
\end{aligned}
$$


where $A, B, C$, and $D$ are constants that may be determined by applying the boundary conditions of (24).

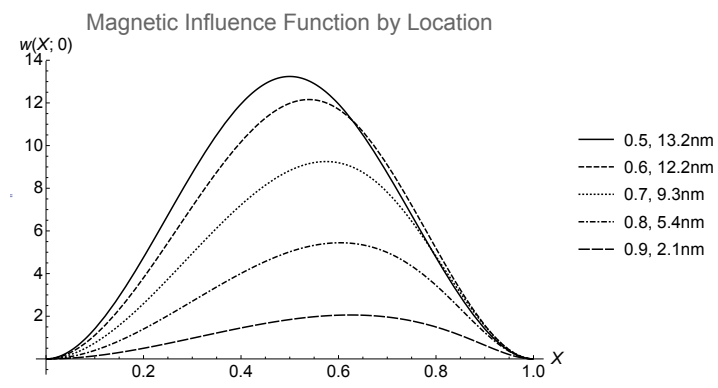

(a)

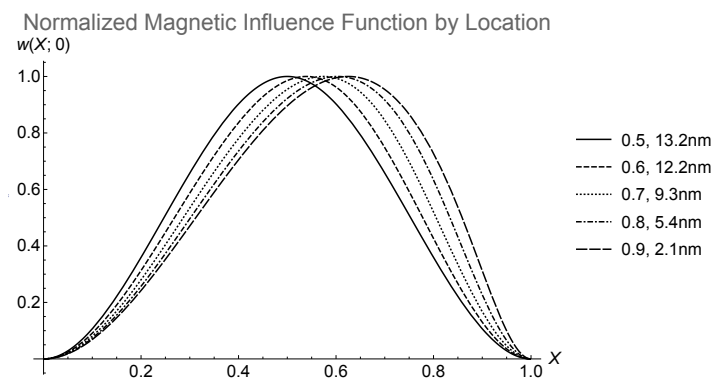

(b)

Figure 6: The analytical facesheet deflection $w(x ; 0)$ due to an electromagnet as determined by $(32)$ is shown for actuator locations $x_{i}=\{0.5 a, 0.6 a, 0.7 a, 0.8 a, 0.9 a\}$. The facesheet profile is plotted in (a) dimensional form and (b) normalized form. The dimensions used are given in Table 2.

This solution is shown in Figure 6 parameterized by actuator location. In reaching these results, (32) was evaluated using Wolfram Mathematica. We observe that the influence functions due to magnetic actuation are not well localized, and that the magnitude of actuation is strongly dependent on the position of the actuator, decreasing closer to the edge of the mirror. This result is not surprising because the facesheet is modeled as a plate with zero strain in plane of the neutral surface. We also observe that the peak of the influence function for an actuator located at $x=0.9 a$ is at approximately $x=0.7 a$, implying that only approximately the central $40 \%$ of the aperture diameter is useful for control. In order to improve the ferrofluid DM design based on the solely on the results of this model, the facesheet must clearly be made thinner in order to lower the flexural rigidity. As the facesheet is made less rigid however, the assumptions outlined in $\S 2.1$ must be re-addressed. In particular, to allow for the increased flexibility of the facesheet in the model, the strain in the neutral surface of the facesheet must be considered. In designing an overall thinner facesheet, there is a risk of introducing sensitivity to vibration, lessening the gravitational invariance of the DM in various orientations, and increased initial warping of the facesheet. This latter effect can be measured and included in the model, so long as superposition holds. In $\S 4$ an example is given which illustrates how finite-element simulations are being used to design a flexible facesheet with more localized influence functions without sacrificing the optical surface quality.

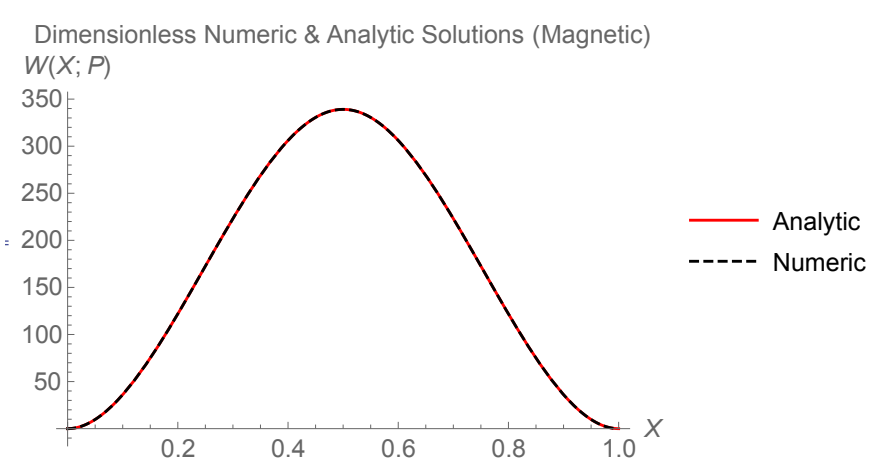

Figure 7: Comparison of the numerical solution to (22) subject to (24) (black dashed line) and the analytic asymptotic solution (32) (red solid line) demonstrates excellent agreement. The solutions were determined for a centrally located actuator at maximum strength $\left(X_{i}=0.5\right.$ and $\left.M_{i}=1\right)$ with no pressure applied $(P=0)$. In calculating these solutions, the same values of the dimensionless parameters presented in Table 2 were used.

To validate the asymptotic approach, the numerical solution to (22) was computed for a prototypical ferrofluid DM with a centrally-located actuator at maximum strength. Figure 7 shows both this numerical solution and the corresponding analytic asymptotic solution (32) to (26) in dimensionless form. Upon comparison to the full numerical solution, we see that the smallness of the aspect ratio $\varepsilon$, which is expected to typify the low-stroke 
regime of space-based wavefront control, causes the asymptotic solution to be a near-exact approximation. The agreement encourages continued application of asymptotic analysis to simplify the high degree of coupling in the ferrofluid DM system, particularly in the extension of the model to the dynamic case and in the improvement of the model by relaxing the facesheet deflection assumptions to allow more flexibility (i.e., strain in the neutral plane).

\section{COMPUTATIONAL MULTIPHYSICS SIMULATIONS}

In this paper we have successfully pursued $1 \mathrm{D}$ analytic solutions for the facesheet deformation in the quasi-static limit of the full coupled dynamic equations. As described in $\S 2$ and illustrated in Figure 2, the next increment of model complexity is to extend to the $2 \mathrm{D}$ case. This will require solving the PDE given by (15) numerically. The coupling of facesheet and the fluid flow field present the greatest challenge in achieving an accurate prediction of the deflection. Computational multiphysics simulations may be employed to improve the model fidelity, but the approach has limited capability in solving the full coupled system because (15) is a fourth-order PDE defining the moving boundary of a fluid domain. Nevertheless, we can leverage the power of computational multiphysics to augment our theoretical model and inform our interpretation of laboratory observations by studying individual aspects of the ferrofluid DM, uncoupled from the system.

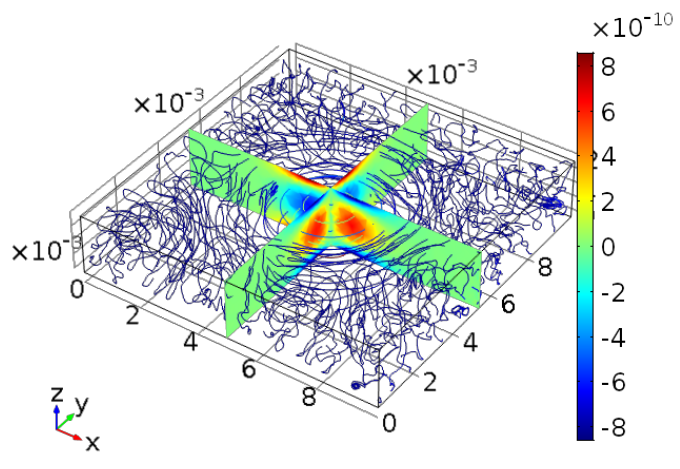

(a) Vorticity $\left[\mathrm{s}^{-1}\right]$

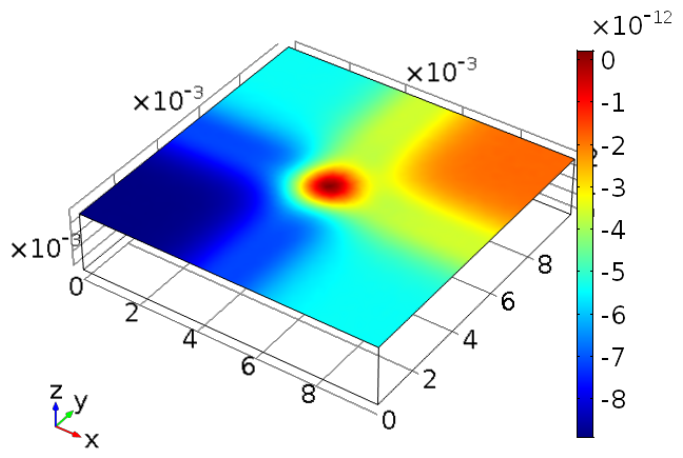

(c) Stress, $z$-component $[\mathrm{Pa}]$

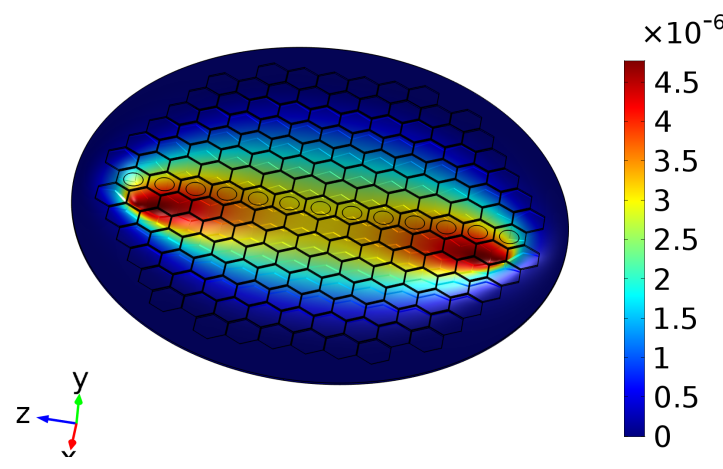

(b) Deflection $[\mathrm{m}]$

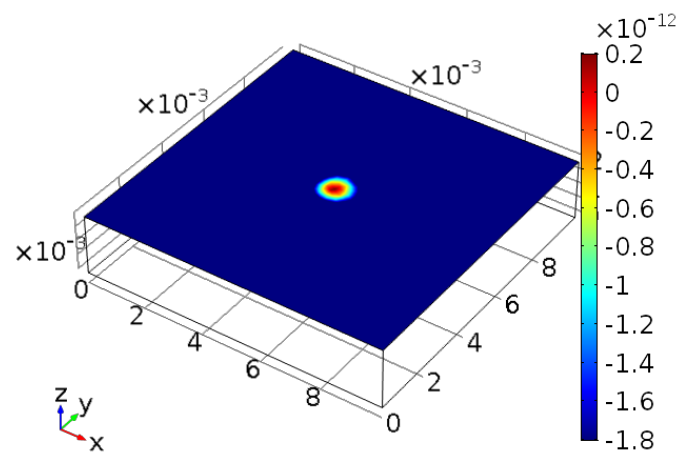

(d) Stress, $z$-component $[\mathrm{Pa}]$

Figure 8: Computational multiphysics simulation of the stress distribution on the facesheet due to a single magnetic coil modeled by (5). Both plots are of the same data, but the dynamic range of (b) has been adjusted to illustrate that a positive stress is centered at the actuator, whereas the rest of the facesheet experiences negative stress. (c) Cross-sections of the steady-state vorticity field and vorticity streamline within a ferrofluid. (d) the deflection of a facesheet with hexagonal back-etched structure to improve actuator localization.

Figure 8 illustrates a few examples of how we are employing COMSOL Multiphysics to improve our physical understanding of the ferrofluid DM and validate our model. The quasi-static model admits a steady-state flow in the ferrofluid, and with COMSOL we can determine and visualize many properties of the flow, such as the vorticity (Fig. 8a). Understanding of the flow field under specific conditions of facesheet deflection, 
actuator current, and ferrofluid pressure can be used to make informed decisions about design changes such as re-positioning the actuators to improve effectiveness or altering the ferrofluid reservoir structure. We have also begun exploring improved facesheet design with COMSOL, such as thinning the facesheet in the vicinity of each actuator with surface-processing techniques in order to create more localized influence functions (Fig. 8b). In the model described above, electromagnetic coils have been idealized as point-source magnetic dipoles with tunable dipole moment. Using a multiphysics package we can calculate the stress on the undeformed facesheet due to this idealized field (Fig. 8c, 8d), and in future work we will compare this to the simulated stress due to a model of the actual coil. In this way it will be possible to computationally determine expressions for the magnetic traction and fluid-magnetic pressure terms on the right-hand side of (15) that accurately represent experimental conditions.

\section{SUMMARY}

In this paper we have presented a 2D dynamic model for the deformation of the facesheet of a ferrofluid DM due to pressure changes and actuation by an array of electromagnetic coils. In the quasi-static 1D case, we found an analytic description of the deflection which may be used to predict the tunable focal length of the DM. We used the smallness of the characteristic facesheet deflection relative to the mirror aperture to derive an asymptotic model of the facesheet deformation under the combined influence of pressure and magnetic actuation. The asymptotic result was shown to be nearly identical to the numerical solution of the full system. The model predicts that the influence function due to a single electromagnet is not well localized and that it is highly dependent on the proximity of the actuator to the edge of the mirror. Any improvement of the facesheet design that increases its flexibility mandates that the model be modified in order to capture these effects.

In addition to the presented theory, we have outlined a plan for incrementally building the model complexity and fidelity. Immediate steps to improve the model theoretically include the admission of lateral and in-plane loads on the facesheet and the inclusion of small initial warping on the facesheet. The first of these can be achieved by modifying (19) to the form

$$
D \nabla^{2} \nabla^{2} w(x)=q(x, w)+N_{x} \frac{d^{2} w}{d x^{2}},
$$

where $N_{x}$ is the in-plane force. A small initial curvature $w_{0}(x)$ may be treated as a 'fictitious' in-plane load, ${ }^{22}$ whereby (19) becomes

$$
D \nabla^{2} \nabla^{2} w_{1}(x)=q\left(x, w_{1}\right)+N_{x} \frac{d^{2}\left(w_{0}+w_{1}\right)}{d x^{2}} .
$$

Additionally, simulations with computational multiphysics software will become an integral aspect of the ferrofluid DM model development. For example, we have begun testing new facesheet designs with improved influence function localization and flexibility, and we have proposed using COMSOL to model the actual electromagnetic coils so as to evaluate the validity of (and/or replace) the dipole field model.

The development of this mathematical model of the ferrofluid DM, represents progress toward realizing a DM with high-precision low-stroke capabilities for high-contrast imaging. Presently, this model is serving to improve our understanding of the physical interplay of key parameters and inform the engineering of an improved ferrofluid DM; eventually, the results of this work may be useful in developing control laws to command the shape of a ferrofluid DM for closed-loop wavefront control in a high-contrast AO instrument.

\section{Acknowledgements}

This research was supported by NASA Grant \#NX15AF28G, the Nancy Grace Roman Technology Fellowship in Astrophysics for Early Career Researchers, and Princeton University's Eric and Wendy Schmidt Transformative Technology Fund. 


\section{REFERENCES}

1. M. Kuzuhara and M. Tamura, et al., "Direct imaging of a cold Jovian exoplanet in orbit around the Sun-like star GJ 504," in Astrophys. J. 774(1), pp. 11-30, 2013.

2. P. G. Kalas and A. Rajan, et al., "Direct imaging of an asymmetric debris disk in the HD 106906 planetary system," in Astrophys. J. 814(1), pp. 32-43, 2015.

3. D. Spergel and N. Gehrels, et al., "WFIRST-AFTA 2015 Report by the Science Definition Team and WFIRST Study Office." Technical report, NASA GSFC, 2015.

4. M. Postman, et al., "Advanced Technology Large-Aperture Space Telescope (ATLAST): A technology roadmap for the next decade." Technical report, Space Telescope Science Institute, 2009.

5. K. R. Stapelfeldt and F. G. Dekens, et al., "Exo-C: a probe-scale space observatory for direct imaging and spectroscopy of extrasolar planetary systems," in Proc. SPIE 9605, pp. 96050T, 2015.

6. S. B. Shaklan, L. Marchen, J. Krist, and M. Rud, "Stability error budget for an aggressive coronagraph on a 3.8 m telescope," in Proc. SPIE 8151, pp. 815109, 2011.

7. L. Pueyo and N. J. Kasdin, "Polychromatic compensation of propagated aberrations for high-contrast imaging," in Astrophys. J. 666(1), pp. 609-625, 2007.

8. A. J. Lemmer, T. D. Groff, N. J. Kasdin, D. Echeverri, and I. R. Cleff, "Technological progress of a ferrofluid deformable mirror with tunable nominal optical power for high-contrast imaging," in Proc. SPIE 9605, pp. $960525,2015$.

9. A. Give'on, B. Kerna, S. B. Shaklan, D. C. Moody, and L. Pueyo, "Broadband wavefront correction algorithm for high-contrast imaging systems," in Proc. SPIE 6691, pp. 66910A, 2007.

10. L. Pueyo, J. Kay, N. J. Kasdin, T. D. Groff, M. McElwain, A. Give'on, and R. Belikov, "Optimal dark hole generation via two deformable mirrors with stroke minimization," in Appl. Opt. 48(32), pp. 6296-6312, 2009.

11. A. Riggs, T. D. Groff, A. Carlotti, N. J. Kasdin, E. Cady, B. D. Kern, and A. Kuhnert, "Demonstration of symmetric dark holes using two deformable mirrors at the high-contrast imaging testbed," in Proc. SPIE 8864, pp. 88640T, 2013.

12. S. A. Corenelissen, P. A. Bierden, and T. G. Bifano, "Development of a 4096 element MEMS continuous membrane deformable mirror for high contrast astronomical imaging," in Proc. SPIE 6306, pp. 630606, 2006.

13. O. Cugat, S. Basrour, C. Divoux, P. Mounaix, and G. Reyne, "Deformable magnetic mirror for adaptive optics: technological aspects," in Sensor Actuat. A-Phys. 89, pp. 1-9, 2001.

14. T. D. Groff, A. J. Lemmer, and A. Riggs, "A new deformable mirror architecture for coronagraphic instrumentation," in Proc. SPIE 9904, in press, 2016.

15. E. F. Borra, "Liquid mirrors," in Can. J. Phys. 73, pp. 109-125, 1995.

16. G. Tremblay and E. F. Borra, "Optical tests of a $3.7 \mathrm{~m}$-diameter liquid mirror: behavior under external perturbations," in App. Opt 39(31), pp. 5651-5662, 2000.

17. W. L. H. Shuter and L. A. Whitehead, "A wide sky coverage ferrofluid mercury telescope," in Astrophys. J. Lett. 424, pp. L139-L141, 1994.

18. R. Ragazzoni and E. Marchetti, "A liquid adaptive mirror," in Astron. Astrophys. 283, pp. L17-L19, 1994.

19. A. Iqbal and F. Ben Amara, "Modeling of a magnetic-fluid deformable mirror for retinal imaging adaptive optics systems," in Int. J. Optomechatronics 1(2), pp. 180-208, 2007.

20. E. F. Borra, "Magnetic liquid deformable mirrors for astronomical applications: active correction of optical aberrations from lower-grade optics and support system," in Astrophys. J. Suppl. Ser. 201(2), p. 8, 2012.

21. D. Yogev and S. Efrima, "Novel silver metal liquid-like films," in J. Phys. Chem. 92(20), pp. 5754-5760, 1988.

22. S. Timoshenko and S. Woinowsky-Krieger. Theory of Plates and Shells. $2^{\text {nd }}$ ed. McGraw-Hill, New York, 1964.

23. G. L. Pollack and D. R. Stump. Electromagnetism. Addison Wesley, San Francisco, California, 2002.

24. R. E. Rosensweig. Ferrohydrodynamics. Dover Publications, Mineola, New York, 2014. 